\section{Vielfalt schmeckt und schützt}

ahrzehntelang herrschte in der Frage, wann bei Säuglingen Beikost eingeführt werden soll, die Meinung vor, dass die frühe Gabe die Entstehung von Allergien fördert, die späte hingegen präventiv wirkt. Nach neueren Studien hat die Verschiebung der Beikosteinführung auf den Zeitraum nach dem sechsten Lebensmonat aber keinen präventiven Effekt, sie kann das Risiko für Allergien sogar erhöhen. Seit März 2009 liegt nun die überarbeitete AWMF-Leitlinie zur Allergieprävention vor (Allergo J 2009; 18: 332-41). Diese empfiehlt ausschließliches Stillen in den ersten vier Lebensmonaten und Beikosteinführung im fünften und sechsten Lebensmonat.

Ein innovatives Beikostkonzept mit einem Mehrwert für die Ernährung von Babys bieten die Produkte von Nestlé NaturNes. Darin sind nicht nur Biozutaten verarbeitet - die Nahrung ist zudem konsequent frei von Zucker- und Salzzusätzen, süßenden Saftkonzentraten oder Verdickungsmitteln. Die Rezepturen sind exakt auf Babys Bedürf-

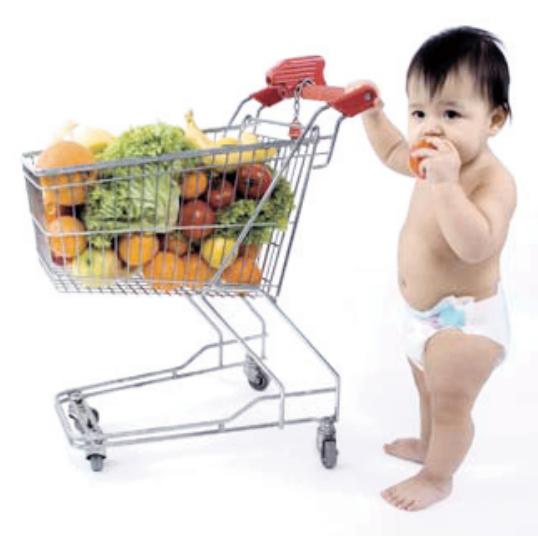

Babys Einkaufswagen sollte bunt gefüllt sein mit Produkten ohne künstliche Zusätze.

nisse in jeder Altersstufe abgestimmt und entsprechen damit den neuesten wissenschaftlichen Erkenntnissen.

„Wir wissen heute, dass sich die Geschmacksvorlieben eines Menschen zu einem großen Teil bereits im ersten Lebensjahr herausbilden. Deshalb ist es wichtig, in diesem Alter auf eine natur- belassene, gesunde Ernährung zu achten“, betont Dr. Mathilde Kersting vom Dortmunder Forschungsinstitut für Kinderernährung. „Mit einer geschmacklich variablen Beikost können die Geschmackspräferenzen der Kinder langfristig positiv geprägt werden". Daher ist es wichtig, Babys möglichst früh eine vielfältige Beikost zu geben.

Bei der Herstellung der NaturNesProdukte werden zwei Prozessschritte kombiniert. Zunächst erfolgt eine schonende Dampfgarung der einzelnen $\mathrm{Zu}$ tatengruppen. Anschließend macht eine Ultrahocherhitzung die Nahrung in nur wenigen Minuten haltbar. Im Vergleich zu herkömmlichen, wesentlich länger dauernden Sterilisationsprozessen ist die Gesamthitzeeinwirkung somit besonders gering. Das schützt die Nährstoffe und den natürlichen Geschmack.

Das Sortiment umfasst Früchte, Gemüse und Menüs. Insgesamt stehen 20 Rezepturen für die Fütterungsstufen 1 bis 3 zur Verfügung.

Nach Informationen von Nestlé Nutrition, Frankfurt/Main

\section{Belastungsasthma bei Kindern reduziert}

U nter körperlicher Belastung verspüren Kinder mit Asthma häufig Symptome, auch wenn sie mit einem inhalativen Glukokortikoid gut eingestellt sind. Wie eine aktuelle Studie zeigt, kann die zusätzliche Gabe von Montelukast $\left(\right.$ Singulair $\left.{ }^{\circledR}\right)$ diesen Patienten zuverlässiger helfen als die Behandlung mit einem lang wirksamen $\beta_{2}$-Agonisten.

Die $\beta$-Agonisten sind zwar in der Lage, belastungsinduzierten Asthmaattacken vorzubeugen, es gibt jedoch Hinweise, dass der bronchoprotektive Effekt in der Dauertherapie mit der Zeit abnimmt. Klinische Vergleichsstudien mit Erwachsenen konnten belegen, dass bei Montelukast die Wirkung nicht nachlässt. Ein internationales Forscherteam hat nun in einer randomisierten doppelblinden Cross-Over-Studie untersucht, ob dies auch für Kinder gilt. Die Ergebnisse wurden im Rahmen des Kon- gresses der European Respiratory Society im September 2009 in Wien präsentiert: 154 Jungen und Mädchen zwischen sechs und 14 Jahren waren für die Untersuchung rekrutiert worden. Sie erhielten nach einer vierwöchigen Run-in-Phase zusätzlich zu einer Fluticason-Basismedikation, die während der Studie unverändert blieb, entweder $5 \mathrm{mg}$ Montelukast oder zweimal täglich $50 \mu \mathrm{g}$ Salmeterol sowie jeweils ein entsprechendes Plazebopräparat über vier Wochen. Nach einer 14-tägigen Auswaschphase wurde die Medikation gewechselt und für weitere vier Wochen verabreicht. Ein Belastungstest wurde während der Run-in-Phase wie auch nach Abschluss der jeweils vierwöchigen Behandlungsphasen absolviert - sechs Minuten auf dem Laufband bei $50-70 \%$ der maximalen Herzfrequenz in den ersten zwei sowie bei $80-90 \%$ in den folgenden vier Minuten. Bei allen Studi- enteilnehmern erfolgten standardisierte Bestimmungen der forcierten Einsekundenkapazität $\left(\mathrm{FEV}_{1}\right)$.

Montelukast führte zu einer besseren Bronchoprotektion als Salmeterol mit einem signifikant geringeren Abfall der FEV1 bei Belastung (10,6 vs. 13,8\%, $\mathrm{p}=0,009)$. Ein Abfall der $\mathrm{FEV}_{1}$ um mindestens $10 \%$ trat häufiger bei Kindern unter Salmeterol als unter Montelukast auf. Auch bei den anderen vordefinierten Endpunkten wie beispielsweise der Zeit bis zur Erholung war der Leukotrienantagonist dem lang wirksamen $\beta_{2}$-Agonisten überlegen. Sowohl Montelukast als auch Salmeterol erwiesen sich als gut verträglich.

Das Fazit der Autoren: Die Zugabe von Montelukast verbesserte bei Kindern unter Fluticason-Basismedikation den Schutz vor Belastungsasthma bei guter Verträglichkeit signifikant wirksamer als Salmeterol.

Nach Informationen von MSD, Haar 